\title{
Oncogenes: 20 years later
}

\section{Introduction}

Nearly two decades have passed since definitive proof was obtained for the proposal that normal cells harbor cancer genes. The fact that normal cellular genes have the potential to become oncogenic leads to the concept of the proto-oncogene, the "enemy within." A defense against cancer cells requires an understanding of normal cells. In the last decade there has been an explosion of information on the nature of the growth signals, the mechanisms by which diverse signals are received by the cell, and the exquisitely streamlined relay system that carries these messages to the nucleus to activate the transcription of genes involved in cell growth, differentiation, and development. The discovery of tumor suppressor cells and their roles as negative regulators of cell growth has created a counterbalance to the growth stimulatory functions of proto-oncogenes. The close ties and interdependency between cell cycle regulation and the growth control genes is beginning to yield insights into the molecular mechanisms of oncogenesis. Cancer research has become a genetic science, invigorated by the convergence of seemingly unrelated fields in molecular genetics and development. It is now common for yeast geneticists, fruit fly developmental biologists, cell fate mappers in worms and mammalian embryologists to share a common platform with cancer biologists. This remarkable confluence underscores the evolutionary conservation of fundamental biological themes. Progress is also being made in transferring the recent advances in fundamental cancer research to clinical applications that provide novel and effective therapies.

This Keystone Symposium ${ }^{1}$ brought together a diverse group of scientists who discussed basic and applied aspects of growth control and of differentiation. The hope was to distill an enormous amount of information into useful knowledge.

The meeting was dedicated to the memory of Dr. Howard Temin, whose pioneering discovery of reverse transcriptase laid the foundation for the oncogene field. The highlights of each meeting session are summarized by the chairpersons in this section. Following the meeting summary are the texts of two keynote addresses- "A Tribute to Howard Temin" by David Baltimore (Massachusetts Institute of Technology, Cambridge, MA) and "Cancer: Emergence of the Genetic Paradigm" by J. Michael Bishop (University of California, San Francisco).

\section{Src: key to kinases and receptors}

G. Steven Martin (University of California, Berkeley)
As reviewed in Michael Bishop's address, the Src gene has played a key role in the development of molecular oncology. This session focused on the Crk protein as a prototype of the non-receptor tyrosine kinases, on the receptor-tyrosine kinases, many of which use Crk as a signaling element, and on the cytoplasmic serine/threonine and dual-specific kinases which convey activating signals from the cell surface to the nucleus.

The Src protein contains a myristoylation domain at the $\mathrm{N}$ terminus. Between the myristoylation domain and the catalytic (tyrosine kinase) domain lie unique, $\mathrm{SH} 3$ and $\mathrm{SH} 2$ domains. In the c-Src protein the $\mathrm{SH} 2$ domain is involved in an intramolecular interaction with the C-terminal phosphotyrosine residue, and this interaction inhibits the catalytic activity of the protein. In viral or activated Src this interaction is disrupted, and overexpression of the activated protein leads to transformation. Thus myristoylation, $\mathrm{SH} 3$ and $\mathrm{SH} 2$ domains all promote transformation. As Steve Martin described, the myristoylation domain can be substituted by a C-terminal membrane-targeting sequence derived from Ras, while the $\mathrm{SH} 3$ and $\mathrm{SH} 2$ domains can promote transformation even when translocated to the C-terminus of the protein. These and other findings suggest that these domains function in cis to target the catalytic domain to substrates involved in transformation.

The SH2 domain of c-Src can undergo an intermolecular interaction with phosphotyrosine residues in receptors such as the PDGF receptor. This interaction results in the activation of $\mathrm{c}-\mathrm{Src}$, and since expression of a $\mathrm{ki}$ nase-inactive mutant of Src block signaling, it appears that the catalytic activity of c-Src is necessary for mitogenic signaling by these receptors. Tony Hunter (The Salk Institute, San Diego, CAl and Sara Courtneidge (EMBL, Heidelberg, Germany) have observed that expression of certain c-Src SH3 mutants can inhibit PDGF mitogenic signaling. This dominant negative effect suggests that the SH3 domain of c-Src is required for signaling by this receptor, perhaps because when c-Src is bound to the receptor via its $\mathrm{SH} 2$ domain the $\mathrm{SH} 3$ domain is free to interact with some downstream effector. Overexpression of c-Myc can overcome the block resulting from overexpression of a kinase-inactive mutant of Src, suggesting that the effects of Src kinase activity may be mediated by Myc.

One possible effector of c-Src is FAK, the focal adhesion kinase. When cells are allowed to attach to fibronectin, FAK is activated, presumably as the result of some integrin-mediated signal and undergoes autophosphorylation at Tyr 397. The SH2 domain of c-Src binds to the FAK tyrosine autophosphorylation site. Tony Hunter re-

\footnotetext{
1"Oncogenes: 20 years later," Keystone Symposium held January 5-11, 1995, at Keystone, Colorado, was organized by Inder M. Verma and Peter K. Vogt.
} 
ported that the binding of c-Src to FAK results in phosphorylation of FAK at Tyr 925. This creates a binding site for the SH2 domain of the adaptor protein GRB2, which as a result becomes complexed to FAK. Adhesion of fibroblasts to fibronectin results in activation of MAP (mitogen activated protein) kinase, probably because translocation of GRB2 to the membrane activates the sos/Ras signaling pathway. Thus anchorage-dependent growth may result, at least in part, from integrin-dependent activation of the MAP-kinase pathway.

Another possible effector of c-Src is p68, a protein which becomes tyrosine phosphorylated at mitosis, both in normal cells and in cells expressing activated c-Src, and which physically associates with c-Src via the Src SH3 domain. c-Src is known to be activated at mitosis, and these observations have suggested that c-Src may play a role at mitosis. Sara Courtneidge's group has examined the role of Src family kinases in cell cycle progression by microinjecting proliferating cells with an antibody directed against the C-terminal domain of Src; this antibody inhibits the activity of both Src and other members of the Src kinase family. Microinjection of the antibody leads to a block at the $\mathrm{G}_{2} / \mathrm{M}$ transition. Microinjection of a Src-specific antibody also leads to $a G_{2} / M$ block in cells genetically deficient in active Fyn and Yes, suggesting that these kinases play redundant roles in regulating the $\mathrm{G}_{2} / \mathrm{M}$ transition.

The receptor tyrosine kinases are activated by binding of specific ligands to the extracellular domain (Fig. 1). Binding of ligand leads to receptor dimerization, intermolecular autophosphorylation and binding of signaling proteins via their SH2 domains. The fibroblast growth factor (FGF) receptor resembles other receptor tyrosine kinases in that it dimerizes on binding FGF. However FGFs are monomeric and bind to their receptors with 1:1 stoichiometry. Joseph Schlessinger (New York University, New York/ reported that the oligomerization of the FGF receptor results from the binding of FGF to the sulfated proteoglycan heparin, which allows multivalent binding of the FGF-heparin complex to several receptor molecules. Schlessinger also reported that the adaptor protein Shc contains at its $\mathrm{N}$-terminus a novel phospho-

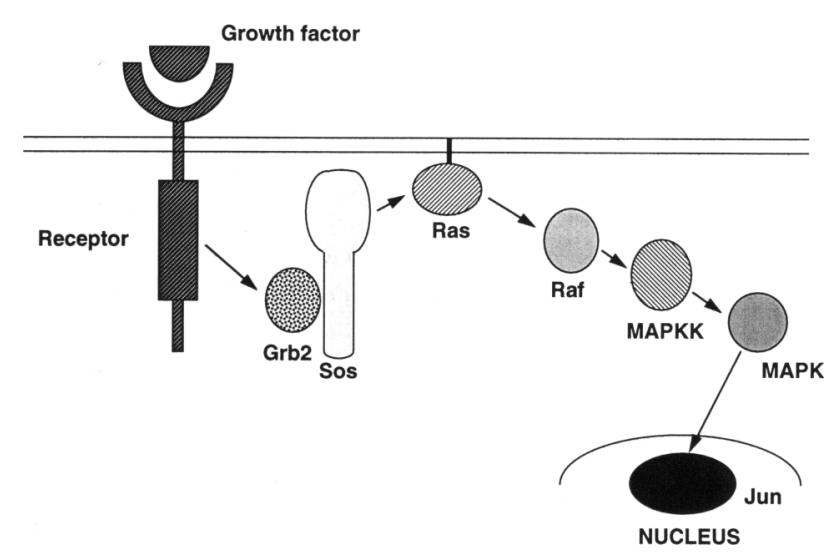

Figure 1. A generalized scheme of signal transduction icourtesy of Dr. Joseph Schlessinger|. tyrosine-recognition domain distinct from the $\mathrm{SH} 2$ domain. This domain binds to a specific motif in the PDGF receptor that is distinct from the site recognized by the Shc SH2 domain.

The signals resulting from the activation of cell surface tyrosine kinases are transmitted to the nucleus by cytoplasmic kinases such as the MAP-kinases. MAP kinase is activated by phosphorylation at threonine and tyrosine by MEKs (the MAP kinase kinases). Mekl is itself activated by phosphorylation at Ser218 and Ser222 by the Rafl kinase. Ray Erikson (Harvard University, Cambridge, MA) and George Vande Woude (National Cancer Institute, Bethesda, MD) have shown that Mekl is constitutively activated by mutation of the serine phosphorylation sites to acidic residues. The specific activity of the double acidic mutants was similar to that of wild-type Mek 1 activated by Raf 1 in vitro, and injection of the mutant protein into Xenopus oocytes induced MAP kinase activation and germinal vesicle breakdown.

During oocyte maturation and meiosis the c-Mos protein is required for the activation and maintenance of MPF, the maturation promotion factor $/ \mathrm{cdc} 2 / \mathrm{cyclin} B$ complex); in this pathway Mos may act upstream of Mek. Chromatin condensation and spindle formation are believed to result from activation of MPF. However Vande Woude reported that under conditions where MPF activation is blocked, both Mos and Mek can induce chromatin condensation and spindle formation, albeit at a slower rate than in the presence of MPF, suggesting that these enzymes can directly induce the early stages of meiosis. Furthermore in somatic mammalian cells, overexpression of c-Mos leads to abnormal mitoses in which the spindles are without asters and are juxtaposed to the cell membrane. These abnormal spindles resemble meiotic spindles, which are attached to the oocyte membrane during polar body formation. The abnormal mitoses which occur in cells overexpressing c-Mos lead to the production of binucleate cells and genetic instability. Thus the transforming activity of c-Mos may result from the inappropriate expression of its meiosis-inducing activity.

\section{Cytoplasm: the connectors}

\section{Joan Brugge (Ariad Pharmaceuticals, Cambridge, MA)}

This session focused on molecular interactions that are responsible for coupling proteins involved in intracellular signal transduction. Most of the presentations included studies of $\mathrm{SH} 2$ and $\mathrm{SH} 3$ domains, which are the best-characterized protein domains involved in proteinprotein interactions. Joan Brugge led off the session with a discussion of the binding properties of $\mathrm{SH} 3$ domains. Using a series of biased and unbiased phage display libraries, she and colleagues have explored the binding preferences of the SH3 domains from Src, Fyn, Yes, Lyn, $\mathrm{Abl}, \mathrm{PI}-3 \mathrm{~K}$, and Grb-2. These studies indicated that SH3 binding sequences could be separated into "core" sequences, which were common to many of the $\mathrm{SH} 3 \mathrm{do}$ mains and "flanking" sequences, which show unique 
specificity for each SH3 domain. With the exception of $\mathrm{Abl}$, all of the SH3 domains examined selected a common core peptide sequence with the consensus motif, Arg-Pro-Leu-Pro-Pro- $\xi$-Pro. Using this peptide to construct biased libraries, this group examined binding preferences of Src, Fyn, Yes, Lyn, PI-3K, and Grb-2 SH3 domains in the region flanking the amino and carboxyl terminus of these core sequences. The studies indicate that binding selectivity of $\mathrm{SH} 3$ domains is at least in part determined by amino acid sequences flanking the $\mathrm{NH}_{2}$ and $\mathrm{COOH}$-terminus.

David Baltimore continued with the theme of SH3 binding ligands. Using $\lambda$ gt 11 phage screening, his laboratory isolated a cDNA that contained a seven amino acid sequence that binds to the $\mathrm{SH} 3$ domain of Src with high affinity (Arg-Pro-Leu-Ala-Leu-Pro). Cotransfection of cells with c-src and a GST fusion gene containing a portion of this cDNA caused an increase in transcription of a reporter gene known to be activated in v-Src transformed cells. The GST-SH3 ligand fusion protein is phosphorylated on tyrosine under these conditions. These results raise the possibility that a SH3 ligand can activate c-Src through interactions with the SH3 domain. Using the yeast two-hybrid system, CRAF-1, a gene whose product binds tightly to the intracytoplasmic tail of the $\mathrm{CD} 40$ protein, was isolated. This protein consists largely of four domains: a C-terminal region that bind to CD40 and is homologous to the TNF $\alpha$ receptor binding protein, TRAF; a long amphipathic helix structured as a "isoleucine zipper"; a series of unique zinc fingers; and a RING finger. A clone consisting mainly of the TRAF homology domain acts as a dominant negative for signaling CD23 upregulation through CD40. Thus, CRAF-1 is part of a new family of signaling molecules used by the TNF $\alpha$ R family of receptors. Baltimore also presented evidence that $3 \mathrm{~T} 3$ cells transformed by various oncogenes have reduced levels of caveolin and caveolae. Caveolae are flask-like invaginations of the plasma membrane coated with material rich in caveolin that are thought to participate in signaling. In Abl-transformed cells, caveolin and its mRNA are undetectable and in cells transformed by an Abl temperature-sensitive mutant, caveolin mRNA and protein is increased by a shift to the nonpermissive temperature. Thus, caveolin down-regulation may be an important new aspect of the transformed state.

Tony Pawson (Mt. Sinai Hospital, New York) summarized the evidence indicating that $\mathrm{SH} 2$ domains have different binding specificities. In particular, he compared the structure of the Src and C-terminal SH2 domains of PLC- ${ }_{\gamma 1}$. The former binds preferentially to pTyr-GluGlu-Ile, and possesses two pockets, one for pTyr and a second hydrophobic pocket for the Ile. The PLC- ${ }_{\gamma 1} \mathrm{SH} 2$ domain, in contrast binds preferentially to hydrophobic residues following the pTyr, and has extended hydrophobic groove that accommodates these residues. $\mathrm{He}$ emphasized the point that $\mathrm{SH} 2$ domains do more than simply bind activated receptors, and noted that the $\mathrm{Src} \mathrm{SH} 2$ domain has multiple functions including a proposed intramolecular interaction with its own $\mathrm{C}$-terminal tail that inhibits kinase activity. He then showed NMR data indicating that the Crk SH2/SH3 adapter protein such an intramolecular SH2-pTyr interaction can in fact be directly identified. Pawson noted that a second pTyr binding (PTB) domain on the Shc protein is conserved between Drosophila and mammalian Shc, and provided evidence that it recognizes NPXpY motifs. Thus, unlike $\mathrm{SH} 2$ domains, which show strongest preferences for sequence carboxy-terminal to pTyr, PTB domains appear to bind pTyr in the context of amino-terminal residues. Lastly, Pawson discussed the functions of $120^{\text {Ras-GAP. }}$. He described evidence that GAP is required for mouse embryonic development, for correct formation of the vasculature, and for preventing aberrant cell death in the developing nervous system. Biochemically, GAP appears to be specifically required for downregulating GTP Ras after growth factor stimulation.

Beatrice Knudsen, from Hidesaburo Hanafusa's laboratory (The Rockefeller University, New York), focused on structural and functional studies on the Crk family of adapter proteins (v-Crk, c-Crk-I, c-Crk-II, and CRKL) which consist predominantly of $\mathrm{SH} 2$ and $\mathrm{SH} 3$ domains. In cells transformed by the c-Crk oncoprotein, the $\mathrm{v}$-Crk SH2 domain binds to at least three tyrosine phosphorylated proteins $\mathrm{pl} 30$ (CAS), a protein that corresponds to the preferred $\mathrm{pY}-\mathrm{X}-\mathrm{X}-\mathrm{P}$ motif for $\mathrm{Crk} \mathrm{SH} 2$ domain binding, AFAP-110, an actin filament-associated Src substrate and paxillin, a constituent of focal adhesions plaques. The Crk SH3 domain was found to bind to two guanine nucleotide exchange factors, SOS and C3G, the kinases c-Abl and c-Arg, and several additional, yet unidentified proteins of $185 \mathrm{kD}, 145-155 \mathrm{kD}$, and $85 \mathrm{kD}$. Binding of c-Abl to N-terminal Crk SH3 domain results in phosphorylation of the c-Crk-II protein in the spacer region between the two $\mathrm{SH} 3$ domains. This phosphorylation generates a high affinity binding site for the Crk $\mathrm{SH} 2$ domain. In addition, the endogenous cytoplasmic, tyrosine phosphorylated c-Crk-II remains uncomplexed suggesting that the phosphorylation of $\mathrm{c}$-Crk-II by c-Abl may negatively regulate c-Crk-II binding activity. Since the truncation of $\mathrm{c}$-Crk eliminates the tyrosine residue, c-Crk forms constitutive complexes with $\mathrm{c}-\mathrm{Abl}$ and other binding proteins, suggesting an involvement of c-Abl in v-Crk transformation. C3G, a guanine nucleotide exchange factor, contains four proline-rich sequences in the middle of the protein which are capable of binding individually to the $\mathrm{N}$-terminal Crk SH3 domain. Alignment of these sequences with sequences in other Crk SH3-binding proteins pointed to conserved positively charged amino acids C-terminal of the P-X-X-P SH3-binding motif. Further structural information of this unusual lysine-specific interaction was obtained from the crystal structure of the Crk SH3 domain complexed to the high affinity peptide. In the crystal, the lysine residue in the peptide was tightly coordinated by three acidic residues in the SH3 domain. In contrast, the co-crystal structure of the Crk SH3 domain and a peptide containing an arginine at the equivalent position revealed non-optimal geometry for the arginine.

Art Weiss (University of California, San Francisco) pre- 
sented an overview on the protein interactions that play a critical role in $T$ cell receptor (TCR) signaling. The TCR initiates signal transduction events that lead to cellular protein tyrosine phosphorylation. The TCR CD3 and $\zeta$ chains interact with two families of cytoplasmic PTKs via sequence motifs termed immunoreceptor tyrosine activation motifs (ITAMs), containing the consensus sequence YXXLA (6-8) YXXL. The PTKs of the Src family, Lck or Fyn, and of the Syk/ZAP-70 family interact with the TCR ITAMs in a coordinated and sequential manner to initiate activation. Based on studies of an Lck deficient mutant $\mathrm{T}$ cell line and of a heterologous Cos cell system in which mutants of Lck and ZAP-70 were expressed, Weiss proposed that the following model by which the TCR initiates PTK activity: Stimulation of the TCR results in oligomerization of ITAMs, resulting in their tyrosine phosphorylation by Lck. If both tyrosines within an ITAM are tyrosine phosphorylated, ZAP-70 is recruited via both of its $\mathrm{SH} 2$ domains to a doubly phosphorylated ITAM. This allows for the interaction of Lck and ZAP-70 which leads to ZAP-70 tyrosine phosphorylation and to a synergistic activation of TPK activity. This synergistic induction of PTK activity depends upon ZAP-70 catalytic function, suggesting an important role for ZAP-70 kinase activity in cellular protein tyrosine phosphorylation. This latter notion is further supported by their recent observations of a severe combined immunodeficiency disease syndrome which appears to be the result of mutations in the ZAP-70 coding sequence.

Ursula Klingmüller, from Harvey Lodish's laboratory (The Whitehead Institute, Cambridge, MA), described the role of SH-PTP1 in the erythropoietin (EPO) receptor signaling pathway. She showed that the protein-tyrosine phosphatase, SH-PTP1, associated via its $\mathrm{SH} 2$ domains with the tyrosine-phosphorylated EPO-R. In vitro binding studies identified phosphorylated tyrosine $(\mathrm{Y}) 429$ in the cytoplasmic domain of the EPO-R as the site of SHPTP1 interaction. When co-expressed with JAK2 in insect cells, SH-PTP1 specifically dephosphorylates and inactivates JAK2. This suggests that activation of SH-PTP1 binding to the EPO-R plays a major role in terminating proliferative signals.

\section{Cytoplasm: the connectors II}

James E. Darnell (The Rockefeller University, New York)

This session continued the theme of intracellular signaling events and discussed novel pathways. Jim Darnell outlined the newly recognized JAK-STAT pathway and summarized new evidence that phosphorylated tyrosines on receptor chains are the docking sites for STAT proteins, which themselves become phosphorylated by JAKs on tyrosine (Fig. 2). In addition, evidence based on domain swaps between Statl and 3 indicated that DNA binding domain of STATs lies within residues 400-500. Full activation of Statl as a transcriptional activator in response to interferon- $\gamma$ was shown to require serine

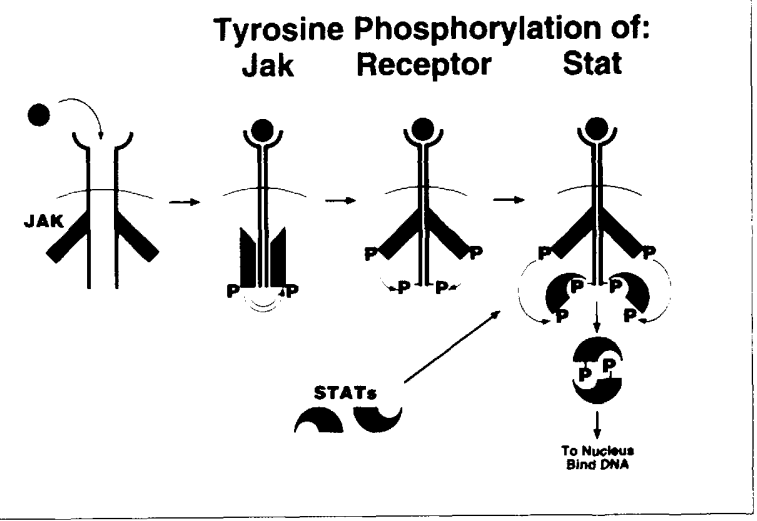

Figure 2. Scheme for JAK-STAT pathway of signal transduction (courtesy of Dr. James Darnell).

phosphorylation on residues 727 in addition to the previously recognized tyrosine phosphorylation on residue 701.

Frank McCormick (Onyx Pharmaceuticals, CA) summarized work showing that Ras proteins activate a protein kinase cascade that begins with Raf and results in activation of MEK and the MAP kinases ERK1 and ERK2. The role of Ras in the process is to recruit Raf to the plasma membrane. Thereafter, Raf activation is Ras independent. This pathway promotes DNA synthesis, and is necessary for Ras-driven oncogenesis. However, Ras oncoproteins have additional effects on cells. Specifically, they cause profound changes in cellular architecture and membrane morphology. These effects are known to be mediated by proteins of the Rac and Rho family. McCormick and colleagues tested the hypothesis that Ras activation of the Rac and Rho pathway contributes to oncogenic transformation. A dominant negative mutant of Rac (N17Rac) blocked focus formation by Ras12. Similarly, a dominant negative mutant of MEK blocks Ras dependent transformation. Ras therefore needs two signaling pathways for full transformation. To investigate the contribution made by $\mathrm{Rac}$ in the process of transformation, they made lines expressing activated Rac constitutively. These cells were found to be fully transformed by the criteria of focus forming activity and tumor formation in nude mice. They also showed that Rac and Rho pathway synergizes with the Raf pathway to transform cells. McCormick concluded that Ras activates at least two independent pathways that act synergistically to give the full effect of Ras malignant transformation. A kinase called PAK65 is activated by Ras and may represent another pathway potentially involved in Rac signaling and thus in Ras transformation.

Doug Lowy (National Institutes of Health, Bethesda, $\mathrm{MD}$ ) discussed negative regulation of Ras by neurofibromin, the product of the neutrofibromatosis (NF1) gene, which is mutated in type 1 neurofibromatosis. Neurofibromin has homology with the catalytic domain of RasGAP and possesses Ras-specific GTPase activating pro- 
tein (GAP)-like activity in vitro. His talk emphasized that the biochemical effects of neurofibromin on Ras appear to differ in a cell type-specific manner. Neurofibromin is an important GAP of Schwann cells, which are a major target of type 1 neurofibromatosis. Human schwannoma cell lines from patients with type 1 neurofibromatosis and mouse Schwann cells derived from embryos that are homozygous for loss of NF1 have low to absent levels of neurofibromin and a high proportion of GTP * Ras. An interesting, but incompletely understood, biological observation is that Ras stimulates the growth of schwannomas but slows the growth of primary Schwann cells. In contrast to Schwann cells, low-to-absent levels of neurofibromin in melanoma and neuroblastoma cell lines from non-FN1 patients are not correlated with high levels of GTP ${ }^{\star}$ Ras, nor is there a significant reduction in GTP* Ras in NIH-3T3 fibroblasts that overexpress neurofibromin sufficiently to slow the growth of the cells. The growth inhibition by neurofibromin in NIH-3T3 is still at the level of Ras, probably by competing for $\mathrm{GTP}^{\star}$ Ras with positive downstream targets.

Gerry Rubin (University of California, Berkeley) showed that the sevenless protein tyrosine kinase receptor plays a central role in the pathway of cell fate induction that determines the development of the R7 photoreceptor in the Drosophila eye. Using a genetic approach, Rubin and colleagues have begun to dissect the signal transduction pathway that relays information from the sevenless kinase. The results support a model in which the stimulation of Ras activity is a key element in signaling by sevenless. A decrease in the level of the products of Ras1 or Sos, which encodes a putative guanine nucleotide exchange factor, or Drk, which encodes a SH3-SH2-SH3 protein, reduces the efficiency of signal transduction. In contrast, mutations of the Gap1 gene, which encodes a putative GAP, increase signal transduction. Moreover, expression of a constitutively activated Ras1 protein bypasses the requirement for the sevenlessmediated signal. These investigators have carried out a large-scale screen for mutations that either increase or decrease the effectiveness of signaling by activated Ras 2 during eye development. Among the genes identified as decreasing signaling are the Drosophila homologs of Raf, MapKK, and MapK. Two transcription factors whose activities appear to be directly regulated by MAPK phosphorylation were also identified-Pointed, a positive factor whose activity is increased by phosphorylation, and Yan, a negative factor that is inactivated by phosphorylation. The screen also led to the isolation of mutations in the genes encoding subunits of the Ras modification enzyme GGT and the phosphatase PP2A, as well as mutations in a gene encoding a novel proteins, phyllopod, that is required in only a subset of photoreceptor precursors and whose expression appears to be induced by ras activation. Perhaps the most intriguing gene discovered in this screen encodes a novel protein kinase that, based on genetic data, acts downstream of Ras, but upstream of or in parallel with Rat

Tom Roberts (Dana-Farber Cancer Institute, Boston,
$\mathrm{MA}$ ) described his studies on polyoma middle $\mathrm{T}$ antigen (MT) and its association with cellular proteins. Over a dozen such proteins are now known, all of which appear to be important in signaling. These include at least three members of the src family of tyrosine kinases, two subunits of PI 3 kinase, two subunits of protein phosphatase 2A, ShC, Grb2, PLC ${ }_{\gamma}$ hsp70, and several members of the 14-3-3 family. Data was presented to show that: (1) PP2A plays the same formal role for MT as a ligand does for a receptor tyrosine kinase, i.e., PP2A binding is essential for $\mathrm{MT}$ to bind and activate src family tyrosine kinases. When MT is mutated so that it can no longer bind PP2A, MT fails to find pp60c-src and to transform. (2) With PP2A removed, a cryptic binding site for hsp70 is revealed on MT. Hsp70 binding is not essential for MT function, but the hsp 70 binding site is conserved in large $\mathrm{T}$ and may play a key role in large $\mathrm{T}$-mediated viral DNA replication. By analogy with prokaryotic systems, hsp70 may also be required for cellular replication. (3) Once MT has used PP2A to activate src kinases, it is phosphorylated on several tyrosines. One tyrosine, 315 , forms a binding site for the SH2 domain of PI3 kinase. Mutants in a novel motif NPXY abolish transformation, but can be rescued by inserting a second NPXY motif elsewhere in the same MT molecule. When phosphorylated, the tyrosine in the NPXY motif serves as a binding site for SHC. SHC binds this NPXY site not via its SH2, but by a novel domain discovered by others, terms PTB or PID. The NPXY motif of MT is not essential for binding PI3 kinase in vitro, but is essential for MT to activate PI3 kinase in vivo. This suggests that GTP loaded p21 ras may be necessary locally for PI3 kinase activation. (4)

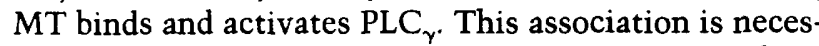
sary for transformation under conditions of growth in low serum.

\section{Nucleus: the responder}

\section{Inder M. Verma (The Salk Institute, San Diego, CA)}

Nuclear oncoproteins that are induced in response to external signals and have the potential to act as transcription factors were the focus of this session. Inder Verma discussed the regulation of the NK- $\mathrm{kB} /$ Rel family of proteins which are present in the cytoplasm of an inactive state in association with $I_{\kappa} B$ protein. Upon induction of Pre B cells with LPS, TPA, or TNF, the I $k B \alpha$ protein is rapidly degraded, followed by the appearance of NK- $\mathrm{kB} /$ Rel activity in the nucleus. He showed that I $\kappa \alpha$ protein present in the NF- $k B /$ Rel complex in the cytoplasm is phosphorylated at its $\mathrm{C}$-terminus by casein kinase II. Addition of TNF $\alpha$ to HeLa cells leads to further phosphorylation of $\mathrm{I}_{\kappa} \mathrm{B} \alpha$. The hyperphosphorylated form of $I_{\kappa} B \alpha$ remains associated with the $\mathrm{p} 50 / \mathrm{p} 65$ complex and presumably is a clue for its degradation. Verma also showed that I $k B \alpha$, in which CKII phosphorylation sites are mutated, has a longer half-life and acts as transdominant negative for NF- $k B /$ Rel activity. Finally, it was shown that during preB to $B$ cell differentiation, the composition of NF- $\mathrm{kB} /$ Rel proteins is altered and constitu- 
tive NF- $\mathrm{kB} / \mathrm{Rel}$ activity in mature B cells is due to rapid degradation and synthesis of IKB $\alpha$.

David Baltimore described the physiological role of the p50 subunit of the NF- $\mathrm{kB}$ complex by targeted disruption of NFKB-1 gene. Mice lacking the p50 subunit show no developmental abnormalities, but they do exhibit multifocal defects in immune responses involving $B$ lymphocytes that include: (i) no proliferation in response to bacterial lipopolysaccharide; (ii) defective in basal and specific antibody production; (iii) inability to effectively clear infection with Listeria monocytogenes, presumably reflecting a defect in macrophage activation; (iv) increased susceptibility to infection to Streptococcus pneumoniae; $(\mathrm{v})$ increased resistance to infection with murine encephalomyocarditis and mengoviruses. The remarkable result here is that there is so little effect upon removal of p50 and that there is no compensatory increase in the amount of other complementary NF- $\mathrm{kB}$ / Rel proteins like p52, Rel, RelB, p65, etc. These are subtle defects and require very careful analysis to underscore defects.

Continuing on the theme of targeted disruption of NF$\kappa B /$ Rel family members, Rodrigo Bravo (Bristol Myers Squibb, NY) described the consequences of a germ line mutation of RelB gene. These mice showed: (i) Absence of RelB protein and dramatic reduction of constitutive $\mathrm{KB}$ binding activity in thymus and spleen; (ii) phenotypic abnormalities that include mixed inflammatory cell infiltration in several organs, myeloid hyperplasia, splenomegaly due to extra hematopoiesis, and a reduced population of thymic dendritic cells; and (iii) impaired cellular immunity. Bravo concluded that RelB function is crucial for both the immune and hemopoietic systems and, more importantly, cannot be compensated for by any other member of the NF-kB/Rel family. Another noteworthy point was that, like the p50-/- mice, the RelB - / - mice also had no developmental defects. Because dorsal is closely related to the NF- $\mathrm{kB} / \mathrm{Rel}$ family members and has a crucial role in the dorsal-ventral polarity of Drosophila, it was often thought that NF- $\mathrm{kB}$ / Rel proteins may have a role in development. The presently available data from the "knock-out" mice described by Baltimore and Bravo clearly dispel this notion.

Peter Vogt (The Scripps Research Institute, La Jolla, CA) described the various domains of v-jun and c-jun proteins required for trans-activation and cellular transformation. He also elaborated on the many structural changes between $v$-jun and c-jun that may impart to $\mathrm{v}$-jun a higher transforming and tumorigenic potential. He made the provocative observation that in chicken cells, there may be no direct correlation between the ability of Jun proteins to trans-activate transcription and cause cellular transformation. He further reported that overexpression of nuclear factor 1 (NF1) in chicken embryo fibroblasts protects the cells from transformation by nuclear oncogenes, but leaves them susceptible to transformation by cytoplasmic oncogenes. All nuclear oncogenes code for transcription factors that are widely assumed to convert signals received from cytoplasmic oncogenes into patterns of gene expression that deter- mine the oncogenic phenotype. One intriguing explanation of the paradoxical NF1 effect is that nuclear oncoproteins are not obligatory components of the transforming signals that emanate from the cytoplasm and that transformation by cytoplasmic oncogenes is independent of the oncogenic potential of nuclear oncogenes.

The intricate network of transcriptional activation and repression mediated by the MYC/MAD/MAX family of basic helix-loop-helix (bHLH) proteins was described by Robert Eisenman (Fred Hutchinson Cancer Research Center, Seattle, WA). MAX is an obligatory partner for myc function, since MYC/MAX heterodimers bind to specific DNA sequence and activate transcription. The MAD protein, on the other hand, can compete with MYC to form MAD/MAX complexes that repress transcription. The levels of MAX mRNA or protein are stable whereas during differentiation of HL60 cells MAD protein is rapidly induced and the MYC:MAX complexes switch to MAX:MAD complexes, which can repress the expression of MYC-regulated genes. In an effort to understand the mechanism of repression by $\mathrm{MAD}$, Eisenman and colleagues have identified two homologs of $\operatorname{Sin} 3$ (mSin $3 \mathrm{~A}$ and $\mathrm{B})$, a repressor protein in $S$. cerevisiae that interacts with MAD protein. The $\mathrm{mSin}$ :MAD association is mediated through one of the four paired amphipathic helix domains in $\mathrm{mSin} 3$ and a potential amphipathic helix located in the $\mathrm{N}$-terminal portion of MAD. Furthermore, the mSin:MAD complex can interact with MAX through the MAD bHLH zip region and bind DNA as a ternary complex. A point mutation within the MAD N-terminal helix disrupts association with MAX by tethering a novel mammalian repressor to a specific DNA binding site. Finally, it was shown that by using the yeast two-hybrid system and MAX as "bait" a number of novel MAX interacting bHLH proteins has been identified. It thus appears that MAX is at the epicenter of MYC/MAD family of transcription factors. To underscore the importance of MAX protein in cellular regulation, Ronald De Pinho (Albert Einstein College of Medicine, The Bronx, NY) mentioned that the consequences of a MAX homozygous "knock-out" mutation are lethal.

Thomas Graf (EMBL, Heidelberg, Germany) described himself as a full fledged member of the "oncogene veterans association" and proceeded to describe his experiences with oncogenic E26 virus containing $M y b$ and ets oncogenes as a model for studying cooperation among oncogenes for transformation of specific cell types. He emphasized the need to identify the relevant target genes of nuclear oncogenes. Using viral ts mutants, he reported the $\mathrm{v}$-myb inactivation in myeloid cells transformed by the oncogenes leads to massive apoptotic cell death in addition to their differentiation. This phenomenon could be linked to the regulation of $\mathrm{v}-\mathrm{Myb}$ of the Bcl-2 gene because expression of the latter depended on V-Myb activity. Furthermore, cell death could be prevented by forced $\mathrm{Bcl}-2$ expression. The fact that v-Myb-transformed myeloid cells require, in addition to Bcl-2, a myeloid-specific growth factor indicates that their survival is controlled by two distinct signals. These observations 
also suggest $\mathrm{Bcl}-2$ as a direct and relevant target of the $\mathrm{v}-\mathrm{Myb}$ oncogene.

\section{Cancer: chromosomes and translocation}

\section{George Klein (Karolinska Institute, Sweden)}

George Klein introduced the session by giving a historical review, focused on the Ig/Myc and the Ig/Bcl-2 translocation. The essential phenotypic consequence of $\mathrm{Ig} /$ Myc translocation in Burkitt Lymphoma (BL) cells is the creation of a "resting cell that is not resting," i.e., a B lymphocyte that has reached the point in its development where it switches phenotypically to a G0-like cell. The fixation of this phenotype not only provides the cell with a continuous drive to proliferate, but also facilitates its escape from immune rejection. Like normal resting B-cells that appear to provide the latent reservoir of persisting virus in healthy individuals, BL cells express only one of the nine growth transformation-associated EBV encoded proteins, EBNAl. Experimental data were presented to suggest that EBNAl is not recognized by immune surveillance, in contrast to the other transformation-associated proteins.

Janet Rowley (University of Chicago, IL) discussed other leukemia-associated translocations. She gave a historical review of the two translocations that were first identified in human malignant diseases: the $t(8 ; 21)$ translocation found in acute myeloblastic leukemia with maturation (AML-M2) and the $\mathrm{t}(9 ; 22)$ translocation that generates the Philadelphia $\mathrm{Ph}^{1}$ chromosome in chronic myelogenous leukemia. Since that time, over 60 recurring translocations have been identified in AML and the breakpoints of almost 30 of these have been cloned. With one exception, all translocations create fused genes, encoding chimeric proteins consisting of the $5^{\prime}$ portion of one gene fused in frame to the $3^{\prime}$ portion of the partner gene. She summarized our current understanding of the molecular consequences of two translocations involving the AML1 gene at $21 \mathrm{q} 22$ and the TEL gene at $12 \mathrm{pl3}$, which is likely to be a transcription factor.

Ronald Evans (The Salk Institute, San Diego, CA) spoke about retinoic acid receptors (RAR) in human disease. The balanced $t(15 ; 17)$ translocation is exclusively associated with acute promyelocytic leukemia (APL), and is often the only visible karyotypic aberration present in this disease. Accordingly, this translocation has become the definitive marker for APL. The translocation in APL involves reciprocal fusion between the RAR and a gene (PML) that encodes a novel nuclear protein. The translocation is proposed to create an oncogene whose product, PML-RAR, is suggested to be a chimeric transcription factor. Evans has shown that PML is a molecular marker for a novel nuclear body referred to as the PML oncogenic domain or POD. The POD is present in normal cells but disrupted in APL. Immunohistochemistry demonstrated that the wild-type POD is composed of at least four other nuclear proteins that associate to form the surface of a large macromolecular structure. In leukemic cells from APL patients, the POD is disrupted into a microparticulate pattern as a consequence of the expression of the PML oncoprotein. Retinoic acid treatment of APL cells triggers a reorganization of PML to generate normal-appearing PODs followed by cellular differentiation. The POD partially dissociates during the normal $S$ phase of the cell cycle. This suggests a potential role for one or more POD-associated proteins in cell cycle progression. Also the POD undergoes a dramatic morphological change during adenovirus infection. It appears that adeno-replication (and transcription) requires one or more proteins in the POD and that the adenovirus E4 product promotes release of a critical factor from the complex.

Michael L. Cleary (Stanford University, CA) presented data about Hox genes and their regulators in lymphoid neoplasia. Two chromosomal translocations in B-cell precursor leukemias involve the E2A gene, which codes for transcriptional proteins of the basic helix-loop-helix class that are essential for B-cell development. Both translocations fuse E2A with genes coding for heterologous transcription factors, in one case resulting in the synthesis of a chimeric homeodomain protein known as $\mathrm{E} 2 \mathrm{a}-\mathrm{Pbxl}$ and in the other case a chimeric basic leucine zipper protein known as E2a-Hlf. Fusion with E2a alters the transcriptional properties of $\mathrm{Pbxl}$ and Hlf, converting them into potent activator proteins as assessed on synthetic reporter genes. The E2a-Pbxl chimeric protein transforms a variety of cell types, including lymphoblasts in transgenic mice. Wild-type Pbxl is not oncogenic but rather requires fusion with E2a, a feature that distinguishes it from all other oncogenic Hox proteins. $\mathrm{Pbxl}$ is a structural and functional homolog of Drosophila extradenticle, which functions as a cofactor to modulate the DNA-binding properties of Hox/Hom proteins. Structure/function studies suggest that E2a-Pbxl may require interactions with heterologous Hox proteins for its transcriptional and transforming activities. These studies are now beginning to provide the early outlines of a model for how disruption of the regulatory activities of homeodomain proteins may lead to oncogenic transformation of lymphoid cells.

Michael Wigler (Cold Spring Harbor Laboratory, NY) dealt with the mutational activation of the Ras oncogene, known to occur in a large proportion of human cancers. He reviewed the organization of Ras regulated pathways in $S$. cerevisiae, S. pombe, and vertebrates, pointing out that Ras has multiple effector pathways in each. In $S$. pombe, the fission yeast, Ras regulates a protein kinase cascade, similar to the MAP kinase cascade of vertebrates, and a morphogenic pathway that leads to Rho, another small GTPase. Strong evidence was presented that Ras transforms cells by contacting Raf. He also reported the use of RDA (representation difference analysis| to detect loci containing genetic lesions in cancer cells, particularly homozygous deletions and amplifications. Deleted regions were identified by using DNA isolated from pure human tumor cell lines to drive hybridization against matched normal DNA. Applying the method in the reverse way, using normal DNA to drive hybridization against tumor cell DNA, probes were iso- 
lated that can detect amplification. A probe was identified that can detect very frequent homozygous losses in $3 p$ in colon cancer cell lines. Using these two approaches, altogether seven genomic loci were identified that appear to be homozygously lost in different tumor cell lines, and two loci that have undergone gene amplification. Since the size of the homozygous deletions is relatively small (less than $50 \mathrm{~kb}$ ), positional cloning of relevant genes becomes more efficient when the RDA methodology is applied to the detection of DNA losses in tumors, than other techniques used for this purpose.

Owen Witte (University of California, Los Angeles) showed that Bcr-Abl uses multiple independent pathways to signal both the ras and to other pathways essential for transformation. This corrects the prior impression in the literature that only a single interaction of Bcr-Abl through Grb-2 is essential for such activity. Witte also described a genetic complementation scheme that permits the positioning of other downstream genes in a physiological and functional context for transformation with Bcr-Abl. Cyclin Dl was identified as an essential partner in signaling from Bcr-Abl. In a second line of study, he described the details of how Bruton's tyrosine kinase regulates the growth of B lymphocytes. A gain of function was detected in which BTK requires a property enabling it to create a cellular transformed phenotype. This mutation was shown to fall within the pleckstrin homology domain of BTK. This was the first demonstration of a mutation in the pleckstrin homology of any protein which leads to an up-activation of biological activity.

\section{Tumor suppressor genes}

Eric Stanbridge (University of California, Irvine)

Eric Stanbridge introduced this session with an historical overview of the discovery and characterization of tumor suppression and tumor suppressor genes. He discussed possible functional interactions between the protein products of oncogenes and tumor suppressor genes, and also debated whether DNA mismatch repair gene defects may be considered a third class of cancer-causing genetic events. Major issues still left unresolved are the "dominant" action of oncogenes and "dominant-negative" mutants of p53. Data were provided that suggest that activation of ras oncogenes may be neither sufficient nor necessary for maintenance of the tumorigenic phenotype-a finding with implications for cancer therapy.

Robert Weinberg (The Whitehead Institute, Cambridge, MA/ provided a brief history of the cloning of the RB1 retinoblastoma gene and its role in control of the cell cycle. He then focused on recent studies from his group on a cyclic-dependent kinase $(\mathrm{Cdk})$-related kinase, termed MO15. This kinase has been identified as the catalytic subunit of the Cdk-activating kinase, CAK. They now show that a new human cyclin (cyclin $\mathrm{H}$ ) is a MO15-associated protein. Cyclin $\mathrm{H}$ enhances the kinase activity of MO15 towards Cdk2/cyclin A. CAK is made up of two subunits: a kinase subunit (MO15) and a cyclin subunit (cdb1). The yeast homologs of these subunits are kin $28 / \mathrm{ccl}$. The kin 28 protein is a component of the RNA polymerase II complex. Thus, the intriguing possibility exists that CAK may be involved in transcriptional control and the regulation of the cell cycle.

Ed Harlow (Massachusetts General Hospital, Boston) continued on the theme of retinoblastoma and retinoblastoma-associated genes and their protein products. $R B$ plays an important role in progression of the cell cycle and functions as a gatekeeper. As cells transit Gl they reach a restriction point where the metabolic decision to proceed to $S$ phase or not is made. The hyophosphorylated form of $R B$ is the active state. In this state $R B$ inhibits progression through $G 1$ to $S$ and the cells may enter the quiescent G0 state that may herald induction of differentiation or apoptosis. Relief of this block occurs by increasing phosphorylation of $\mathrm{RB}$, a modification that involves cyclins $\mathrm{D}$ and $\mathrm{E}_{\text {; }}$ and their respective cyclindependent kinases (cdk). An added level of control is provided by several cdk inhibitors, including p16 $6^{\text {ink4 }}$, $\mathrm{p} 15^{\text {ink } 4 \mathrm{~b}}, \mathrm{p} 21$, and $\mathrm{p} 27^{\mathrm{kip} 1}$. The $\mathrm{p} 15^{\text {ink4B }}$ and $\mathrm{p} 16^{\text {ink4 }}$ proteins inhibit cdk4/cdk6-cyclin D complexes whereas $\mathrm{p} 27^{\mathrm{kip} 1}$ inhibits $\mathrm{cdk} 2 / \mathrm{cdc} 2$. The $\mathrm{p} 16^{\text {ink4 }}$ has experienced a checkered career in its short history since the advent of its cloning. Just over a year ago it was identified as a tumor suppressor gene (MTS1) found deleted or mutated in many different cancer cell lines. Rapid screening of human tumors quickly raised doubts as to the veracity of this claim. However, more careful analyses have, indeed, documented proof for involvement of this gene in a variety of malignancies. The inhibition by $\mathrm{p} 16^{\text {ink4 }}$ of activation of cyclin D by cdk4/6 results in the active hypophosphorylated $\mathrm{RB}$ and accompanying inhibition of cell proliferation. In human tumors evidence is emerging for an inverse correlation of $\mathrm{RB}$ and $\mathrm{p} 16^{\text {ink } 4}$ status, i.e., wild-type wtRB:mutant pl6 $6^{\text {ink } 4}$ or mutant RB:wt pl $6^{\text {ink4 }}$. Either condition would relieve cell cycle inhibition.

Francis Collins (National Institutes of Health, Bethesda, $\mathrm{MD}$ ) provided an update on the human breast cancer gene, BRCA1. Familial breast cancer is observed in 1/200-1/300 women. Fifty percent of families with inherited breast cancer have BRCAl mutations. BRCA1 mutations lead to a higher risk of ovarian, colon, and prostate cancer in addition to breast cancer. Mutations in the BRCAl gene were screened using SSCP. Many different mutations were identified, some of which appear to be only polymorphisms, although they might have some effect on disease susceptibility. Now that the gene has been cloned, one must consider mutation testing for high-risk individuals. However, many problems are associated with diagnosis of BRCAl mutations. The gene is very large, which will make testing difficult. In addition, it is unclear how these patients should be managed once it is determined that they carry the mutated gene. Genetic counseling for these families is very complex, and the diagnosis of BCRAl mutations may lead to genetic discrimination. Collins also discussed his 
group's studies on the cloning of breakpoints in chronic lymphocytic leukemia (CLL). CLL is characterized by anomalies of chromosome 16, including inversions and balanced translocation involving both copies of chromosome 16. Cloning has demonstrated that these rearrangements have resulted in a fusion gene involving the transcription factor CBFB, which maps to the short arm, and the myosin heavy chain gene which maps to the long arm of chromosome 16. In all cases studied the breakpoint in the CBFB gene occurs at the same place whereas the breakpoint in the myosin heavy chain gene is variable. The fusion product has been shown to function as a transforming oncogene in NIH3T3 cell assays.

Geoff Wahl (The Salk Institute, San Diego, CA) presented evidence for the involvement of p53 and RB in growth arrest following DNA damage, and how compromising that control, for example, mutations in p53 or $\mathrm{RB}$, will lead to genome destabilization. Based upon a variety of data, it is concluded that p53 initiates a long term, if not permanent arrest, in response to as few as one double-strand break, or a small but not yet precisely determined number of single-strand gaps. Thus, a longterm arrest mechanism effectively rids the population of cells with genomes that are damaged, and prevents the outgrowth of cell containing genetic abnormalities such as gene amplification. It is very important that the cell have a mechanism for eliminating cells with just a solitary double-strand break as gene amplification can occur by repeated bridge-breakage-fusion cycles, which would generate just one double-strand break per cell cycle. Furthermore, the sensitivity of the arrest mechanism provides a way by which p53 could be involved in the senescence clock; as telomeres get shorter, perhaps the shortest would resemble a broken end and trigger a p53-dependent arrest mechanism. He also demonstrated that DNA damage alone is sufficient to induce the arrest, and provided evidence that the breaks do not have to reside in a chromosome to induce the arrest. Wahl suggested that damage as discrete as one broken end is probably sufficient to induce a p53-dependent arrest.

\section{The cell cycle}

Jean Wang (University of California, San Diego)

There is some evidence that activators of cdk, for example, cyclins, may have oncogenic potential whereas cdk inhibitors could act as tumor suppressors. This theme was expanded by David Beach (Cold Spring Harbor Laboratory, NY) who reported the cdc25, the dual specificity phosphatase that can dephosphorylate and activate cdk, has oncogenic potential. In mammalian cells, three different cdc25 family members have been identified: cdc25-A, -B, and -C. Beach showed that overexpression of either cdc25-A or -B (but not -C) in mouse embryo fibroblasts (MEF) resulted in endo-reduplication, indicating successive activation of $S$ phase without mitosis. Interestingly, either cdc25-A or -B (but not -C) could collaborate with the activated Ras (G12V) to transform MEF. In another series of experiments, Beach showed that cdc25 can associate with Raf which is a well known downstream target of Ras. Moreover, Raf could phosphorylate and activate cdc25-A and -B (but not -C). In mammalian fibroblasts, Beach showed that cdc25-A and -B co-localized with Raf at the leading edge of plasma membrane upon mitogenic stimulation. The relevant cdk substrate(s) of cdc25-A and -B are currently unknown. Beach also reported the isolation of a cdk 4 point mutant which is amplified in a melanoma cell line. The mutation makes this cdk4 immune to the inhibitory effect of p16. The escape of cdk 4 from p16-mediated inhibition could conceivably contribute to transformation.

Accumulation of the Gl cyclins have been postulated to be the rate-limiting step for $S$ phase entry. Using the tetracyclin-inducible promoter, Steve Reed (The Scripps Research Institute, La Jolla, CA) showed that overexpression of either cyclin D1 or E indeed caused a shortening of G1 by 3 hours in Rat-1 cells. Coexpression of D1 and E cyclins had the synergistic effect of shortening G1 by 9 hours. Chuck Sherr (St. Jude Children's Hospital, Memphis, TN) reported that constitutive overexpression of D-type cyclins has a similar effect on G1 length. Reed also reported the isolation of cyclin $\mathrm{E}$ mutants that are resistant to degradation and identified an important $\mathrm{Thr}$ residue whose phosphorylation may be required for $\mathrm{cy}$ clin E degradation. The cdc/cyclin inhibitor, p27, Reed showed to undergo cell cycle dependent oscillation in that the protein level is high in G1 and $M$ but low in $S$ phase. Sherr showed that 227 could prevent the activation of cdk 4 by CAK and this is the basis for the growth inhibitory activity of cyclic AMP.

Oncoproteins and tumor suppressors are upstream regulators as well as downstream targets of cdk. An important substrate of several cdk/cyclin complexes is RB. David Livingston (Dana-Farber Cancer Institute, Boston, $\mathrm{MA}$ ) reported the interesting link between RB and Mdm2 , an oncoprotein previously known to bind and inhibit the trans-activation function of $\mathrm{p} 53$. $\mathrm{Mdm}-2$ binds the $\mathrm{C}$-terminal region of $\mathrm{RB}$ outside of the $\mathrm{A} / \mathrm{B}$ pocket and it does not bind the RB-related p107 or p130. Overexpression of $\mathrm{Mdm}-2$ can reverse RB-dependent and p53-dependent growth arrest. However, it is not known if Mdm-2 can bind to RB and p53 at the same time. Jean Wang showed that RB can simultaneously bind to E2F and the $\mathrm{c}-\mathrm{Abl}$ tyrosine kinase using its $\mathrm{A} / \mathrm{B}$ and $\mathrm{C}$ pockets, thus $\mathrm{RB}$ contains at least two independent protein binding domains. The $A / B$ and $C$ pockets of $R B$ are regulated by different phosphorylation sites. The growth inhibitory function of a mutant RB, lacking only two phosphorylation sites that inactivate the $C$ pocket, can no longer be suppressed by phosphorylation. A c-Abl mutant that is resistant to $R B$ regulation can antagonize $R B$, further indicating that inhibition of this tyrosine kinase is important for growth suppression. The c-Abl tyrosine kinase can activate transcription and this is correlated with its ability to phosphorylate the $\mathrm{C}$-terminal repeated domain (CTD) of RNA polymerase II. Taken together, Wang proposed that through the phosphorylation of specific sites in $\mathrm{RB}$, a cdk can modulate the RB-assembled E2F/c-Abl complex to regulate the assembly of RNA polymerase 
and the phosphorylation of the CTD, either coordinately or sequentially in the cell cycle. Cyclin and kinases involved in the cell cycle are represented in Figure 3.

\section{The whole animal}

Suzanne Cory (Walter and Eliza Hall Institute, Victoria, Australia)

The power of transgenesis and gene targeting to dissect multistage tumorigenesis was evident in session devoted to whole animal models. It is clear from such studies that although oncogenes can strongly predispose to transformation, tumor onset requires additional mutations. Synergistic mutations can affect other oncogenes, tumor suppressor genes or, only recently appreciated, gene controlling cell survival.

Insertional mutagenesis with retroviruses is an effective strategy for identifying genes capable of synergizing with an oncogenic transgene. Acceleration of lymphoma development in Myc mice, for example, is usually due to proviral insertion near $B m i-1$, which encodes a nuclear zinc finger protein, or Pim-1, which encodes a cytoplasmic serine/threonine kinase. Anton Berns (The Netherlands Cancer Institute, Amsterdam/ has now taken the strategy a step further, using myc transgenic mice devoid of Pim-1. The gene most frequently activated in tumors arising in infected $\mathrm{Myc} / \mathrm{Pim}-1^{-1-}$ mice is Pim-2, presumably because it can functionally substitute for Pim1 . This result suggests that the strategy could be used to identify genes acting in a defined signal transduction pathway. Berns also provided further insight into the somewhat mysterious Bmi-1 protein, which is homologous to the Drosophila protein Psc, a member of the Polycomb group involved in maintaining repression of homeotic genes during development. Intriguingly, disruption of $B m i-1$ results in posterior transformation of

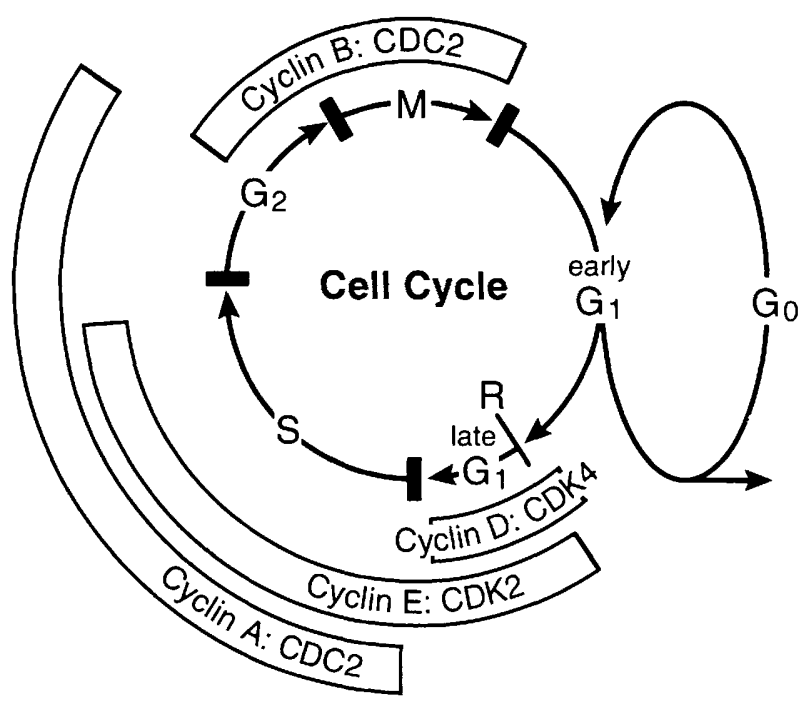

Figure 3. Schematic representation of cyclins and kinases in cell cycle. (Courtesy of Dr. Robert Weinberg.) the axial skeleton, accompanied by a shift in the anterior expression boundaries of certain homeobox genes, while overexpression (via a Bmi-1 transgene) shows the opposite effect. Thus Bmi-1 may regulate expression of specific homeobox genes.

The contrasting roles of Bcl-2 as a savior of cells and wild-type p53 as an executioner were addressed by Suzanne Cory. The $\mathrm{Bcl}-2$ gene, activated by translocation in human follicular lymphoma, encodes a membrane-bound cytoplasmic protein that promotes cell survival in the face of diverse cytotoxic insults. As a transgene, $B c l-2$ only modestly enhances lymphoma onset, but together with $M y c$ becomes a potent lymphomagenic agent. Since $M y c$-expressing lymphocytes die rapidly under sub-optimal growth conditions, the accelerated tumor onset in $\mathrm{Bcl}-2 / \mathrm{Myc}$ mice has been attributed to increased cellular longevity in vivo under conditions of limiting cytokines. The exquisite radiosensitivity of lymphocytes has been attributed to $\mathrm{p} 53$, because its level rises after DNA damage and resting lymphocytes from p53-1- mice are radioresistant. Cory and her colleagues have shown, however, that cycling lymphocytes remain radiation sensitive even in the absence of $p 53$, so DNA damage also evokes a p53-independent path to cell death. Bcl-2 can block both pathways induced by DNA damage. A role for p53 in establishing the "Hayflick" limit was inferred from the finding that transformed hematopoietic colonies derived from p53- / - mice immediately yielded permanent cell lines, whereas those from normal mice died within days. Thus p53 mutation may contribute directly to tumorigenesis by allowing indefinite proliferation as well as indirectly by sparing cells that have undergone mutations as a result of DNA damage.

Doug Hanahan (University of California, San Francisco) outlined multistage transformation in transgenic mice expressing SV40 T antigen in pancreatic islet cells (RIP-Tag mice), highlighting the role of modulation of cell survival. Activation of the insulin-like growth factor II (IGF-II) gene occurs early, suggesting that IGF-II contributes to tumor onset. Consistent with this hypothesis, RIP-Tag mice homozygous for disruption of the IGFII gene develop smaller tumors displaying greater cell death and reduced malignancy. Apoptosis during the early hyperproliferative phase (in transgenic mice that are either wild-type or null for IGF-II) correlates with increased expression of bax, an effector of cell death, while the decreased apoptosis seen in tumors is accompanied by elevated expression of $\mathrm{Bcl}-\mathrm{x}$, a functional homolog of $B c l-2$. Characteristic loss of heterozygosity on chromosomes 9 an 16 suggest that gene inactivation may trigger both onset of hyperproliferation and angiogenesis.

The AP-1 transcription factor is a heterodimeric complex constituted from members of the Fos and Jun families. The challenge is to identify which specific biolog ical processes are critically dependent on individual family members. Studies of mutant mice in Erwin Wagner's laboratory (Research Institute of Molecular Pathology, Vienna, Austria) have shown that c-Jun is vital for liver development and c-Fos for osteoclast-macrophage lin- 
eage determination and bone remodeling. Embryos lacking c-Jun die from impaired hepatogenesis and $c$-/un ${ }^{-1-}$ ES cells can contribute to all tissues in chimeric mice except the liver. By contrast, $c$-Fos ${ }^{-1-}$ mice develop osteopetrosis due to a block in the differentiation and bone-resorbing osteoclasts. Bone marrow transplantation rescued the osteopetrosis and expression of a c-Fos transgene overcame the differentiation block.

The neurofibrosarcoma $(N f-1)$ gene encodes a member of the GAP family of protein that regulate ras. Individuals that inherit a nonfunctional $\mathrm{Nf}-1$ gene are prone to multiple lesions, including benign neurofibromas, and are at increased risk of certain malignancies. Tyler Jacks (Massachusetts Institute of Technology, Cambridge) has reported that mice heterozygous for the mutation are predisposed to various tumor types and a high proportion of the tumors have lost the wild-type $N f-1$ allele. Homozygous deletion is lethal but chimeras generated from Nf-1-null ES cells all develop neurological symptoms and have neurofibromas. These data are strongly indicative of a role for $\mathrm{Nf}-1$ in negative growth regulation and tumor suppression. Jacks has also inactivated the $\mathrm{K}$-ras gene, and unexpectedly, the mutation is an embryonic lethal. In contrast, $N$-ras knockout mice developed in another laboratory are repeatedly viable and normal.

The family of Bcl-2 related proteins is rapidly expanding, as outlined by Stan Korsmeyer (Washington University, St. Louis, MO). Family members share two highly conserved domains implicated in dimerization. Bax heterodimerizes with $\mathrm{Bcl}-2$ and, when in excess, counters the ability of $\mathrm{Bcl}-2$ to repress cell death. Susceptibility to death is best correlated with the relative concentration of Bax in homodimers versus heterodimers, but it is not yet clear which of these complexes is interacting with the death effector pathway. Bax can also heterodimerize with $\mathrm{Bcl}-\mathrm{x}_{\mathrm{L}}$. Significantly, a single amino acid substitution in one of the homology regions of $\mathrm{Bcl}-2$ and $\mathrm{Bcl}-\mathrm{x}_{\mathrm{L}}$ disrupts their ability to heterodimerize with $B a x$ and $a b-$ rogates survival function. A new member of the family, Bad, identified by yeast two hybrid screening and lambda expression cloning, selectively dimerizes with $\mathrm{Bcl}-\mathrm{x}_{\mathrm{L}}$ as well as with Bcl-2. Its affinity for Bcl- $x_{L}$ (but not for Bcl-2) is sufficient to displace Bax and the death repressing activity of Bcl- $\mathrm{x}_{\mathrm{L}}$ (but not that of Bcl-2) is negated. The outcome of a cell death signal is thus influenced by competing dimerizations and the concentration of both $\mathrm{Bad}$ and $\mathrm{Bax}$ will determine whether or not $\mathrm{Bcl}-2$ or $\mathrm{Bcl}$ $\mathrm{x}_{\mathrm{L}}$ can be effective in repressing apoptosis.

\section{The whole animal II}

Janet D. Rowley (University of Chicago, IL)

Continuing with the theme of multistage tumorigenesis, Timothy Lane, from Philip Leder's laboratory (Harvard University Medical School, Boston, MA), presented work on breast cancers that arise in transgenic mice. This laboratory has demonstrated previously that MMTV-Myc constructs result in focal adenocarcinomas of the breast within 200 days. Mating such mice with MMTV-Ha-ras mice carrying a transgene resulted in acceleration of tumor formation in the offspring. Although p 53 mutation are found in $45 \%$ of spontaneous human breast cancers, mice carrying an activated $M y c$ gene and one functional p53 allele showed an increase in lymphomas (all of which showed loss of $\mathrm{p} 53$ ) that were fatal before the possible onset of mammary disease. Infection of MMTVint2/FGF3 transgenic mice with replication-competent MMTV to induce insertional mutagenesis resulted in increased tumor incidence. Insertions clustered near wnt1/ int 1 ; however, an undescribed member of the wnt family (murine Wnt10b) was identified. Unlike Wnt1, Wnt10b is expressed during normal breast development and may regulate mammary epithelial growth and differentiation.

Rudolph Jaenisch (The Whitehead Institute, Cambridge, MA| described the role of DNA methylation in tumorigenesis and raised the intriguing question of whether DNA methylase (DNMT) can act as a mutagen. There are at least two methylases in cells, one involved in maintaining methylation of newly replicated DNA strands and the other required to establish methylation of previously unmethylated DNA. The gene for the former methylase has been cloned; a homozygous deletion of the gene in mice is lethal at the 30-somite stage, whereas embryonic stem cells are viable but have dem. ethylated DNA. If these ES cells are induced to differentiate then they die. The Min mouse which has a chainterminating mutation and truncation of one apc gene, develops about 100 polyps in the intestine within four to six months. Crossing Min with a DNA methyl transferase (Dnmt) heterozygote decreases the number of polyps to only 40 . The Min mouse injected with 5 azadeoxycytidine $\left(5^{\prime} \mathrm{aza}-\mathrm{dC}\right)$, which induces demethylation of DNA has about 12 polyps. The Min/DNMT heterozygote treated with $5^{\prime}$ aza-dC has almost none. If Min animals are treated weekly with 5 'aza-dC, no tumors are seen at 100 days; if $5^{\prime}$ aza-dC is started at day 50 , they have the same number of tumors as untreated controls.

The gene therapy strategy described by Richard Mulligan (The Whitehead Institute, Cambridge, MA) was based on the introduction of the GM-CSF gene into tumor cells using retrovial vectors. The initial studies used irradiated B16 mouse melanoma cells and insertion of the GM-CSF gene, gave $100 \%$ protection against a tumor cell challenge. Although there are no effective strategies against established tumor cells, Mulligan presented some evidence that GM-CSF expression might enhance tumor cell antigen presentation to Langerhans cells. Clinical trials based on this approach are presently underway in renal cell carcinoma and melanoma. One of the problems with the use of bone marrow transplantation is the difficulty in identifying the pluripotent stem cell. Mulligan described a new technique for physically separating stem cells using Hoechst 3342 . He can further enrich for cycling stem cells, which reconstitute irradiated animals with greater efficiency.

Michael Blaese (National Institutes of Health, Bethesda, $M D$ ) is developing direct gene therapy for cancer. His laboratory has used retroviral vectors to transfer two different suicide gene systems into cancer cells: herpes 
thymidine kinase (HS-tk), which phosphorylates the antiviral drug ganciclovir to produce a potent nucleotide toxin, and cytosine deaminase from fungi or bacteria, which converts the benign antifungal drug $5^{\prime}$ fluorocytosine into the highly cytotoxic drug $5^{\prime}$ fluorouracil. In theory, every tumor cell would have to be gene-modified; however, the "bystander effect" results in killing of wild-type tumor cells in the vicinity of a gene-modified cell when exposed to the appropriate product. Brain tumors have been treated using injection of murine fibroblasts producing the HS-tk retroviral vector into multiple sites in the brain tumor and subsequent treatment with systemic ganciclovir. Of 15 patients, about half show some anti-tumor response, and four had variable periods of sustained response. To improve the response, Blaese's laboratory proposes the development of motile vector-producing cells or other viral vectors carrying suicide genes that might replicate specifically within a tumor.

\section{The new frontiers}

\section{Paul Berg (Stanford University, CA)}

Paul Berg reflected on the symposium's revelations and their implications for understanding and therapy of cancer. In contrast to Gunther Stent's admonition (1968) that "the first attempts to write the history of a scientific advance or discipline often presage its imminent senescence," Berg expressed confidence that the 20th anniversary celebration of the discovery of cellular oncogenes and proto-oncogenes demonstrated that the ongoing research was vibrant and increasingly pregnant with clues that are leading to greater understanding of the genetic and molecular aspects of cancer. Hopefully, this will result in a greater likelihood of therapeutic benefits. Given the genetic paradigm of cancer, a primary goal of cancer research remains to identify the genes and functions that are disrupted in tumor cell. This is a difficult challenge given that each cancer may in fact have a different constellation of mutations, each constellation ultimately perturbing normal growth and behavior sufficiently to produce a cancer. Is the phenotype of such a highly mutated cell reversible? Or has the tumor cell progressed to the point of no return? In that case, the most plausible strategy for cancer therapy is to learn how to induce such cells to commit suicide or how to eliminate them, preferentially by chemical or immunologic means.

Tom Curran (Roche Institute of Molecular Biology, Nutley, NJ) presented work on two problems. One focused on the role of $c$-fos expression in neurons of the adult brain as a model to understand how stimulation and transcription may be coupled to account for the complex metabolic and structural changes that underlie memory and learning. Seeking to determine if specific elements of the c-fos promoter were involved in the response to agents (kainic acid) known to induce c-fos transcription and brain seizures, he used mice with transgenes containing variously modified forms of the $c$-fos promoter fused to lac $Z$ and found that all of the $c$-fos promoter enhancer elements were needed to express lac $Z$ following induction of seizures by kainic acid. Curran also reported on the creation of an animal model of the human disease that results from a generalized resistance to thyroid hormones. This was accomplished by a gene knockout of the thyroid hormone receptor $\beta$-gene.

Carol Greider (Cold Spring Harbor Laboratory, NY) reported on the correlation between the presence of telomerase, the RNP polymerase that synthesizes telomere sequences onto chromosome ends and cell immortality. Studies on cell immortalization in culture suggest that telomerase may be required for the growth of immortal cells. Indeed, normal human tissues do not express telomerase activity while it is present in many different human tumors. As a means to analyzing the role of telomerase in cancer, Greider, in collaboration with workers at Geron Corporation, cloned the gene encoding the RNA component of human telomerase. They found that the RNA is expressed in many tissues that do not possess telomerase activity, suggesting that regulation of telomerase production is accomplished at another level, possibly the production of protein moiety. To study that possibility Greider and colleagues purified the Tetrahymena telomerase and then cloned and sequenced the gene encoding telomerase's 95 and $80 \mathrm{kDa}$ subunits. Attempts are continuing to clone the human counterparts.

Harold Varmus (National Institutes of Health, Bethesda, $\mathrm{MD}$ ) reflected on opposing perceptions of the current status and goals of oncogene research. He recited a litany of progress that included the number of oncogenes and tumor suppressor genes now known, our knowledge of several signaling pathways and part of the machinery that controls the cell cycle, the nature of the molecular interactions between proteins and their ligands, DNA targets and other proteins, genetic maps, and technologies for constructing knockouts. Varmus vehemently rejected the view that the field was too mature and lacking in challenges. Instead he focused on the many opportunities that emerged in the symposium's presentations: Nature of the transcriptional targets of oncogenic activity, apoptosis, angiogenesis, cell adhesion, metastasis, and the potential of exploiting our knowledge of oncogenes for furthering analysis of development and neurobiology. He stressed especially the need for applying our basic knowledge of oncogenes to more creative clinical applications, particularly in risk assessment, and evaluation of new and existing drug therapies. But above all, he encouraged a more aggressive exploration of the unexpected, e.g., observations which seem at variance with currently accepted views or which point to new perspectives on cancer.

But Varmus' strong devotion to research showed in his insistence in describing his laboratory's ongoing investigations. One project aims to determine the Wnt (wingless) signaling pathway for determining dorsal-ventral polarity. A second project examined the effect of p53 deficiency on Wnt-1 induced mammary tumorigenesis. He reported that tumors arise earlier (and, in males, more frequently) in p 53-null mice than wild-type mice, an the tumors from p 53 heterozygotes frequently show 
loss of the normal p53 allele. These studies support a critical role of p53 in the progression to malignancy. He also described an experiment in progress to determine if wild-type src and various mutant alleles expressed specifically in osteoclasts can rescue or influence the osteopetrosis that occurs in src knockout mice.

The symposium ended with a caution from Berg, that we must bear in mind that, along with the triumphs, we have also identified large areas of ignorance and that some still unasked questions may lurk beyond our horizons. And, of course, we should be wary, for some things that we now think we know may become less clear in years to come or even prove utterly wrong. 


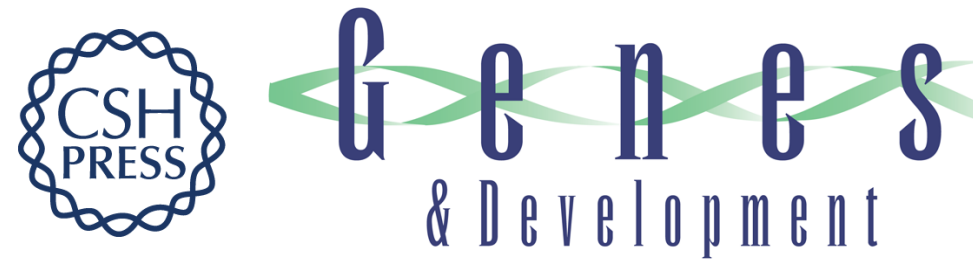

\section{Oncogenes: 20 years later}

Genes Dev. 1995, 9:

Access the most recent version at doi:10.1101/gad.9.11.1289

\section{License}

Email Alerting Receive free email alerts when new articles cite this article - sign up in the box at the top Service right corner of the article or click here.

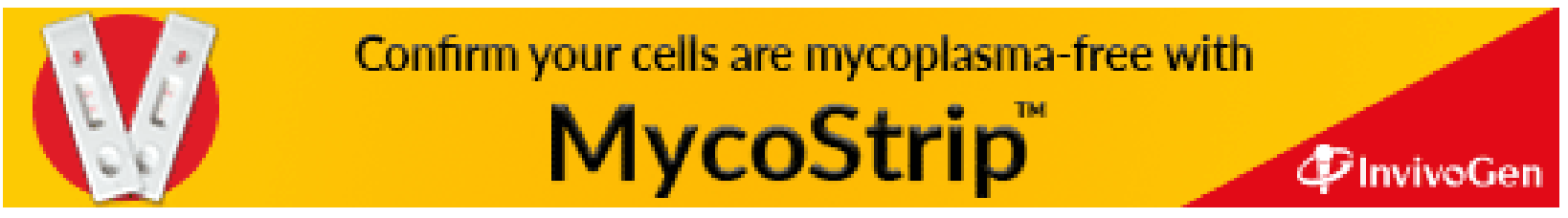

(C) 1995 by Cold Spring Harbor Laboratory Press 